This is the author's final, peer-reviewed manuscript as accepted for publication. The publisher-formatted version may be available through the publisher's web site or your institution's library.

\title{
Turning efficiency prediction for skid steering via single wheel testing
}

D. F. Flippo, D. P. Miller

\section{How to cite this manuscript}

If you make reference to this version of the manuscript, use the following information:

Flippo, D. F., \& Miller, D. P. (2014). Turning efficiency prediction for skid steering via single wheel testing. Retrieved from http://krex.ksu.edu

\section{Published Version Information}

Citation: Flippo, D. F., \& Miller, D. P. (2014). Turning efficiency prediction for skid steering via single wheel testing. Journal of Terramechanics, 52, 23-29.

Copyright: ( 2013 Published by Elsevier Ltd. on behalf of ISTVS.

Digital Object Identifier (DOI): doi:10.1016/j.jterra.2014.02.001

Publisher's Link: http://www.sciencedirect.com/science/article/pii/S0022489814000020

This item was retrieved from the K-State Research Exchange (K-REx), the institutional repository of Kansas State University. K-REx is available at http://krex.ksu.edu 


\title{
Turning Efficiency Prediction for Skid Steering via Single Wheel Testing.
}

\author{
D.F. Flippo ${ }^{\mathrm{a}, 1, *}$, D.P. Miller ${ }^{\mathrm{a}, \mathrm{b}, 2}$ \\ ${ }^{a}$ RM 141 Seaton Hall, Kansas State University, Manhattan, Kansas, United States \\ ${ }^{b}$ The University of Oklahoma, 900 Asp Ave, Norman, Oklahoma, United States
}

\begin{abstract}
Wheel design can be enhanced through experimentation, testing, and iteration. Unfortunately, the time and money needed to test full vehicles is costly. A cheaper, less conflated alternative could be to incorporate single wheel testing. The algorithm discussed in this paper uses sinlge wheel testing to predict the full vehicle performance in a skid steer turn. With this prediction algorithm, skid steering can be easily enhanced by iterating on the design of a single wheel without the cost of vehicle testing. To validate this algorithm and explore skid steering enhancement several single wheel skid steering experiments were done and the results were compared to a full vehicle's turning performance.
\end{abstract}

Keywords: Skid Steer, robotics, testing, wheel to soil, terrain, single wheel testing

\section{Introduction}

Compared to other methods, skid steering has several advantages such as simplicity in design and control which leads to fewer components, less weight,

\footnotetext{
This document is a collaborative effort.

* Principal corresponding author

Email addresses: dkflippo@ksu.edu (D.F. Flippo ), dpmiller@ou.edu (D.P. Miller)

${ }^{1}$ Daniel Flippo received his PhD in Mechanical Engineering in 2009 from the University of Oklahoma, Norman Oklahoma, USA. Currently, he is an assistant professor in the Biological and Agricultural Engineer Department of Kansas State University, USA. His research interests include robotic mobility, Vehicle to Soil Interaction, robotic test beds, robotic development, and off-road vehicles.

${ }^{2}$ David P. Miller is the Wilkonson Chair and Professor in the School of Aerospace and Mechanical Engineering at the University of Oklahoma. He received his $\mathrm{PhD}$ from Yale in 1985 and later initiated the development of small rovers at the NASAs Jet Propulsion Lab., which led to the Mars pathfinder rover mission in 1997. He continues research on planetary surface robotics while also working on other areas of mobile systems including assistive and educational robotics. He was a Co-founder of iRobot Corporation. He is also a Co-founder and Technical Advisor to the KISS Institute for Practical Robotics which provides robotics educations programs in hundreds of schools around the world.
} 
and a more robust vehicle design $<12>$. Because of these advantages skid steering has found many applications in construction, military, and agriculture. Skid steering is not new to the planetary rover field either. The Russian Cold War era rovers Lunokhod I and II used skid steering and were extremely successful in their missions $<9>$.

Skid steering does pose some deterrents as a steering methodology. Predicting the behavior of the vehicle in a skid steer turn is difficult due to the unmeasurable sliding that occurs. Much work is being done currently on analytical solutions for skid steering modeling such as $<10 ; 1 ; 17 ; 16>$. Another deterrent is the low efficiency that results from energy being used to skid the wheels in a turn. Empirical methods can mitigate these disadvantages but full vehicle tests can be expensive and time consuming.

To avoid the costs of full vehicle field tests, we suggest using single wheel testing as part of the iterative design process for improving skid steering. For single wheel tests to be of any use, the data that they generate must have some significance in the real world. The performance in the single wheel testing machine must transfer to predict the behavior exhibited on a multi-wheel vehicle doing typical maneuvers in field conditions. What this paper discusses is the algorithm that allows a transfer to be possible by comparing the predicted vehicle rotation per wheel rotation to that of a vehicle incorporating the same wheels.

\section{Wheel Forces in a Skid Turn}

This paper will focus on a zero radius skid steer turn on a flat hard surface. When a vehicle initiates a turn its rotation (in the $X-Y$ or ground plane) will accelerate up to a certain spin rate $\Omega$ at which point it will stabilize and the moment about its center $\left(M_{o}\right)$ will equal zero simplifying it to a statics problem. Figure 1 shows a top view force body diagram of a vehicle in an equilibrium skid steer turn. The assumptions for the system are:

- other than ground interaction and gravity there are no external forces or accelerations acting on the vehicle.

- Vehicle weight is evenly distributed

- All four wheels rotate at the same rate throughout the test.

- Normal forces are the only loading from suspension to the wheels.

$F_{y}$ is the force along the wheel's spinning direction due to its traction where $f_{y}$ is the opposite force due to the rolling or bulldozing resistance of the wheel. $F_{x}$ is along the wheel's axis and symbolizes any lateral traction induced by the wheel's rotation by its tread, where $f_{x}$ is conversely opposite of $F_{x}$ and is due to the lateral sliding friction of the wheel. $F_{X}$ and $F_{Y}$ symbolize the resultant component forces on the wheel.

$$
\Sigma M_{o}=0
$$




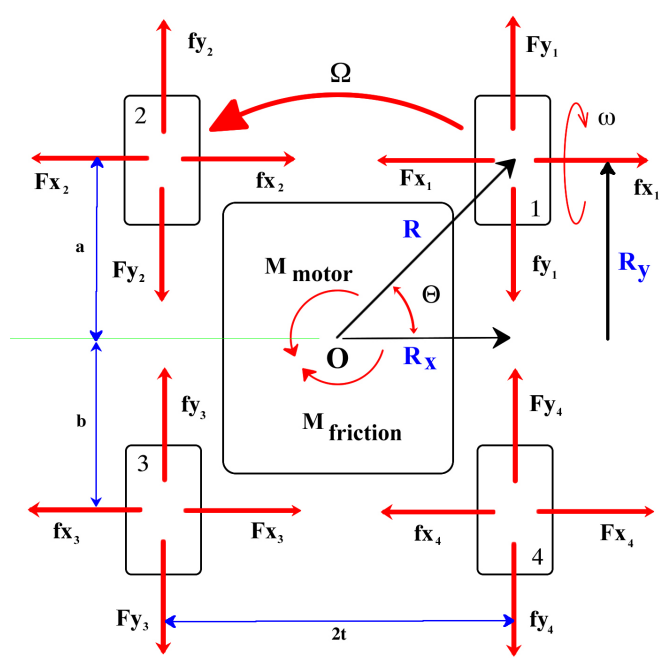

Figure 1: Skid Steer Force Body Diagram

$$
\begin{gathered}
M_{\text {motor }}-M_{\text {friction }}=0 \\
F_{Y}=F_{y}-f_{y} \\
F_{X}=f_{x}-F_{x} \\
\Sigma\left(F_{Y} R \cos (\Theta)\right)-\Sigma\left(F_{X} R \sin (\Theta)\right)=0 . \\
F_{Y}=F_{X} \tan (\Theta) .
\end{gathered}
$$

Equation 6 describes a relationship between $F_{X}$ and $F_{Y}$ at the turning equilibrium point and is dependent upon the vehicle geometry $(\Theta)$. If the vehicle were slender (Figure. 2-a) then $\Theta$ would be larger than $\frac{\pi}{4}$ and $F_{X}$ would be much smaller than $F_{Y}$. If $\Theta=\frac{\pi}{2}$ then $F_{Y}=\infty$. This would mean that no matter how much force a non directional wheel could exert on the ground the vehicle's spin rate $\Omega$ would always be zero. If, on the other hand, $\Theta$ were equal to zero, as in Figure 2-b, then $F_{Y}=F_{y}+f_{y}$ (the net force of power and resistance) would be equal to zero. This configuration is better known as differential steering, or in the case when the wheel orientation can be adjusted to remain tangent to the turn radius is Ackerman steering, originally developed by Erasmus Darwin $<8>$. This means that the wheels have no lateral slip and assuming there is no longitudinal slip then the turning rate, $\Omega$, can be calculated by the following:

$$
V @ w h e e l=\Omega R
$$

As well as:

$$
V_{@} w h e e l=\omega r
$$




$$
\Omega_{\text {Ackerman }}=\frac{\omega r}{R}, F_{Y}=0 .
$$

where $\omega$ is the wheel angular velocity in radians per second, $\mathrm{r}$ is the wheel radius, and $\mathrm{R}$ is the distance from the center of the wheel to the center of rotation of the vehicle (Figure $2 \mathrm{~b}$ ).

Equation 9 refers to the Ackerman turning rate $\Omega_{\text {Ackerman }}$ without longitudinal slipping. To calculate $\Omega$ for a skid steer vehicle $(\Theta \neq 0), \Theta$ must be taken into account and is reflected in Equation 10. $\Omega_{\text {TheoreticalMax }}$ refers to the theoretical maximum a skid steer vehicle can spin, but $F_{Y}$, at $\Omega_{\text {TheoreticalMax }}$, is not zero (Figure 2a).

$$
\Omega_{\text {TheoreticalMax }}=\frac{\omega r}{R} \cos (\Theta), F_{Y} \neq 0 .
$$

To find the value of $\Omega_{F_{Y}=0}$, which is the spin rate at which there is no longer a net force in the $Y$ direction, the longitudinal velocity $\left(V_{y}\right)$ (Figure $2 \mathrm{~b}$ ) of the ground under the wheel must be equal to the velocity of the wheel rim $(\omega r)$ therefore making $F_{Y}=0$ (no slip). Equation 15 explains this relationship.

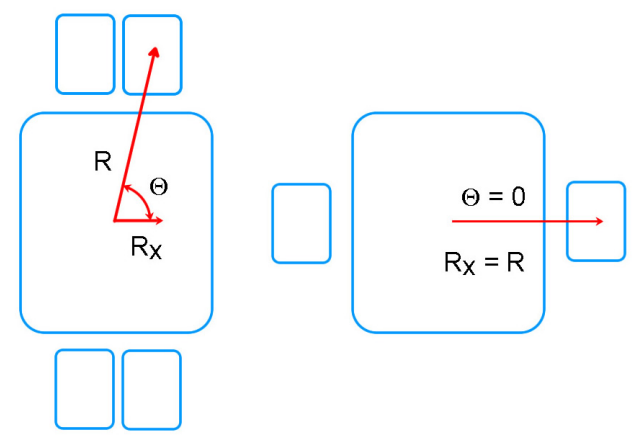

(a)

(b)

Figure 2: Skid steer geometry configurations (a) slender (b) wide

$$
\begin{gathered}
V_{y}=\omega r . \\
V_{y}=\cos (\Theta) V_{\text {ground }} . \\
V_{\text {ground }}=\Omega R . \\
\omega r=\Omega R \cos (\Theta) . \\
\Omega_{F_{y}=0}=\frac{\omega r}{R \cos (\Theta)}=\frac{\omega r}{R_{x}} .
\end{gathered}
$$

For the right front wheel of a vehicle pivoting in the counter clockwise direction, the ground must move under it in the opposite direction $\left(-\Omega \frac{\mathrm{rad}}{\mathrm{sec}}\right)$. The relationship of the forces on the wheel, as the spin rate $(\Omega)$ of the ground under the wheel increases, can be shown in Figure 4 . When the simulated vehicle's 


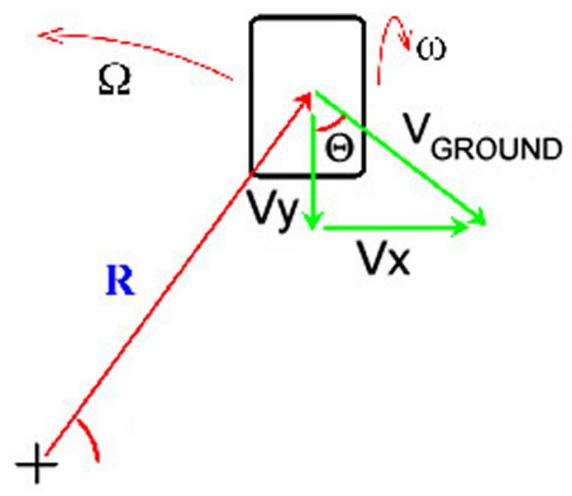

Figure 3: Skid steer kinematics

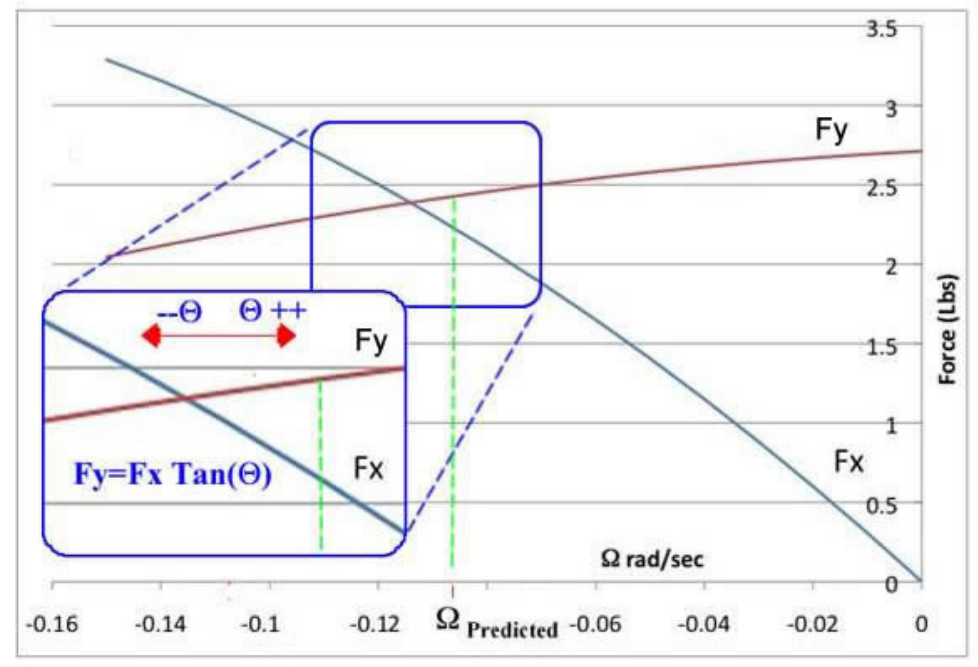

Figure 4: Force vs spin rate example (all forces are total resultant forces $F_{Y}$ and $F_{X}$ ) 
spin rate $(\Omega)$ is equal to zero, the wheel being tested rotates $(\omega)$ but does not move along the ground as if a person was holding the vehicle and obstructing it from spinning. This causes a force in the $Y$ direction which is just the kinetic friction $\left(F_{Y}=\mu_{k} N\right.$ - viscous friction) between the wheel and ground. For a blank wheel on smooth ground there is no $F_{X}$ at $\Omega=0$, but for a directionally treaded wheel $F_{X}$ could be non-zero which will be one value to focus on when testing new wheels. If the person holding the vehicle were to allow it to spin a little faster but still not at its equilibrium spin rate the $F_{X}$ would increase while $F_{Y}$ would decrease. As the vehicle's spin rate is allowed to increase the two forces would continue until they intersect. This meeting point would represent the equilibrium spin rate $\left(\Omega_{\text {Predicted }}\right)$ of a square vehicle $\left(\Theta=\frac{\pi}{4}\right)$. For the SR2 $<12>$ vehicle $\Theta=.8477 \mathrm{rad}$ which when combined with Equation 6 simplifies to the following.

$$
F_{Y}=1.133 F_{X}
$$

In essence what we are doing is operating the wheel and the ground under the wheel independently, while measuring all the the forces and torques involved. The ground speed $(\Omega)$ is increased while keeping the wheel spin rate $(\omega)$ constant. By observing the behavior of the forces acting on the wheel we get a force curve diagram as in Figure 4. When the forces satisfy Equation 16 the corresponding $\Omega$ is the predicted vehicle spin rate. In Figure 4 this relationship gives a point just right of the intersection point and corresponds to a $\Omega_{\text {Predicted }}$ value which is the predicted spin rate of a symmetrically balanced vehicle fitted with four wheels with the same orientation, relative to the vehicle center, and identical tread to the wheel tested.

It should be noted how a vehicle's geometry affects this relationship. As $\Theta$ increases above $\frac{\pi}{4}$ the vehicle is slimmer (Figure. 2) which makes turns less efficient (rate of vehicle turn per wheel rotation) and $\Omega_{\text {Predicted }}$ becomes smaller. If, on the other hand, $\Theta$ decreases its $\Omega_{\text {Predicted }}$ value increases until $\Theta=0$ and $\Omega_{\text {Predicted }}=\frac{\omega r}{R}$ which is a differential steering geometry.

\section{Validation Experiments}

Skid steering turn performance is an example of a typical maneuver, beyond the domain of most single wheel test systems, such as $\langle 2 ; 7 ; 13 ; 14>$, but can be evaluated using the SWEET machine $\langle 5 ; 6\rangle$. The Suspension and Wheel Experimentation and Evaluation Testbed (Figure 5) has a $3 \times 3$ meter footprint and a weighted drop down test leg. A driven wheel and a six-axis, force torque sensor (Figure 11) on SWEET is stationary in the $X$ and $Y$ directions but allows movement along the $Z$-axis via a counterbalance system. SWEET differs from

most testbeds in that the table can move in the $X$ and $Y$ directions underneath the test stand, as well as rotate around an arbitrary point in the $X$ and $Y$ plane. This added advantage gives the apparatus the unique ability to measure forces and torques in a true turn allowing the study of skid steer turning. 


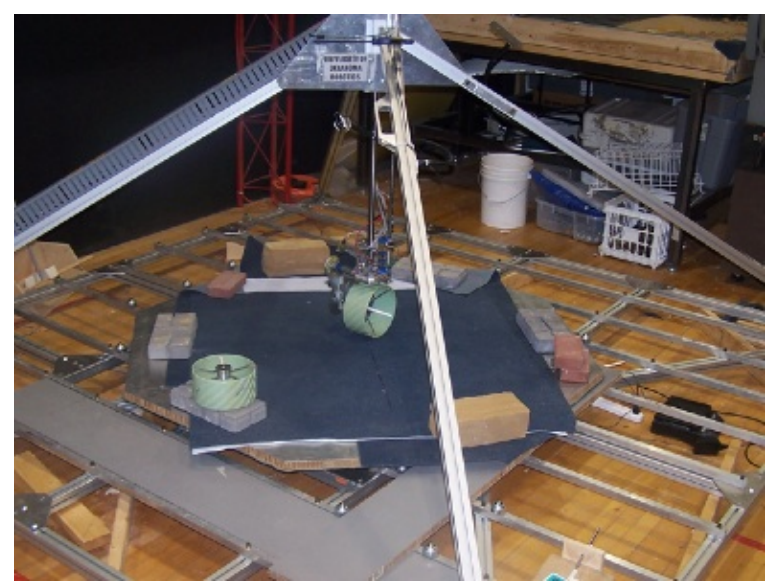

Figure 5: SWEET

SWEET was programmed to simulate a skid steer turn and fitted with a .109 meter diameter blank wheel on simple carpet (Figure 5). Parameters were set to mimic our in-house four wheel skid steer vehicle's (SR2 <12>) geometry and loading. The test variables were wheel spin rates $\left(\omega=.3, .4\right.$, and $\left..5 \frac{\mathrm{rad}}{\mathrm{sec}}\right)$ and turn rates $\left(\Omega=0,-.005,-.01,-.015,-.02 \ldots \ldots .-.10 \frac{\mathrm{rad}}{\mathrm{sec}}\right)$ with 5 trials of each.

The first test set spins the wheel at .3 $\frac{\mathrm{rad}}{\mathrm{sec}}$ and the test table stationary underneath it. SWEET's force sensor measured forces in the $X$ and $Y$ direction in all five trails and then these values where averaged. The next set of trials kept the wheel spin rate constant but increased the table rotational speed to $\Omega$ $=-.005 \frac{\mathrm{rad}}{\mathrm{sec}}$ where the measurements were repeated and averaged. This process was repeated while changing $\Omega$ and leaving $\omega$ constant. All tests were done on SWEET and run as space allowed in its $1 \times 1$ meter test bed. When all these averaged force points are plotted a graph similar to Figure 4 is produced. From these force curves a predicted $\Omega$ is found using Equation 16. The entire process is repeated for $\omega=.4$ and $.5 \frac{\mathrm{rad}}{\mathrm{sec}}$ to find predicted turn rates for that wheel speed.

Post processing of the data, was done with several $\mathrm{C}$ programs that averaged all trials, performed 2nd and 3rd order regression curve fitting, and automatically calculated $\Omega_{\text {Predicted }}$. The results, for this test, are shown in Figures 7,8 , and 9 .

To compare the predicted behavior of these tests to real world behavior an in-house rover $(\mathrm{SR} 2<11>$ ) was used. SR2 (Figure. 6) was fitted with four blank wheels and turned on the same carpet to validate the results. Tests were done for three different wheel speeds $\left(\omega=.3, .4\right.$, and $\left..5 \frac{\mathrm{rad}}{\mathrm{sec}}\right)$ measuring the spin rate of the vehicle during the test (by measuring the angle between an on-board laser level mark and the initial position and dividing by the elapsed time), which 


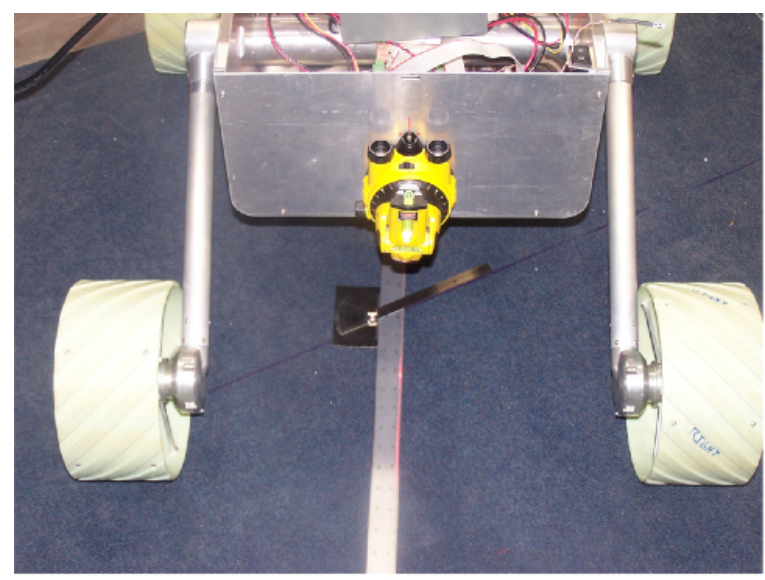

Figure 6: SR2 rover fitted with directional wheels in spin rate validation test

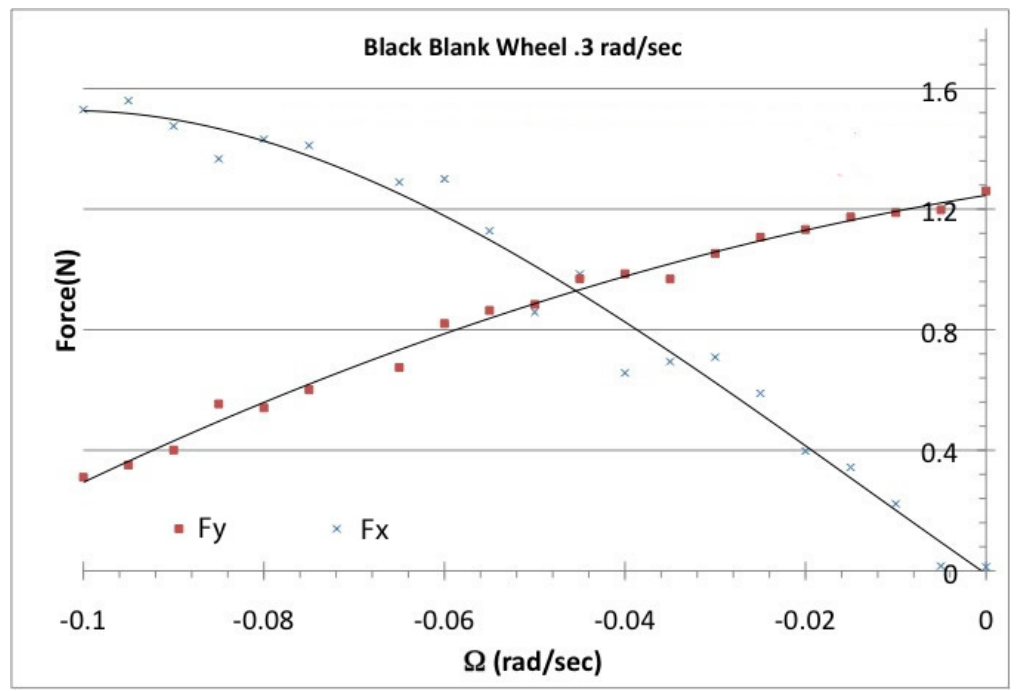

Figure 7: Results for blank wheel at $\omega=.3 \frac{\mathrm{rad}}{\mathrm{sec}}$ 


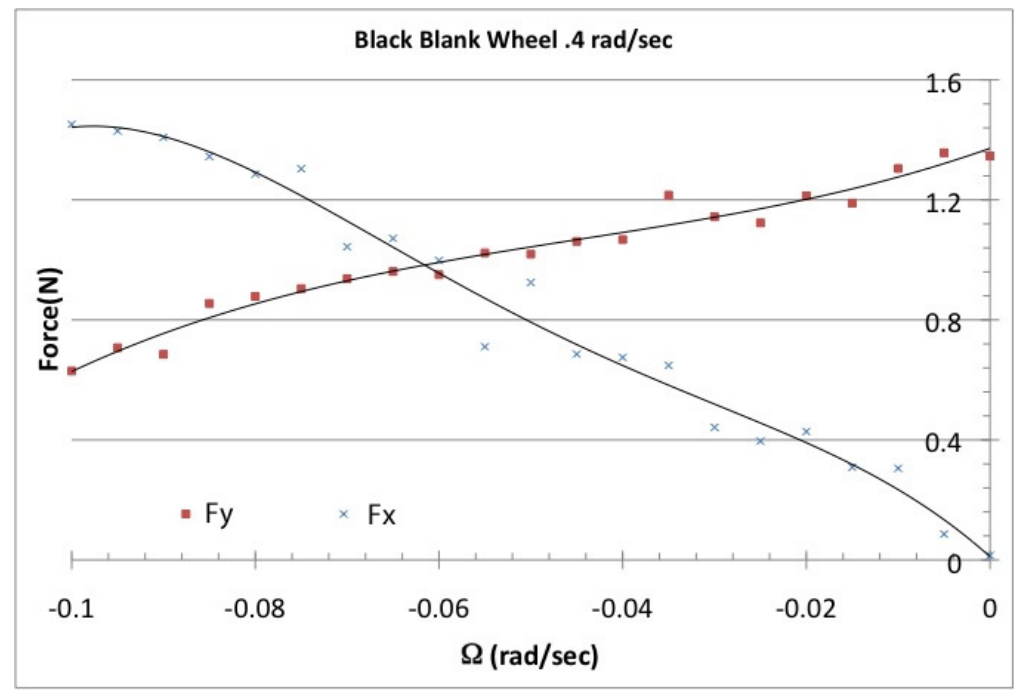

Figure 8: Results for blank wheel at $\omega=.4 \frac{\mathrm{rad}}{\mathrm{sec}}$

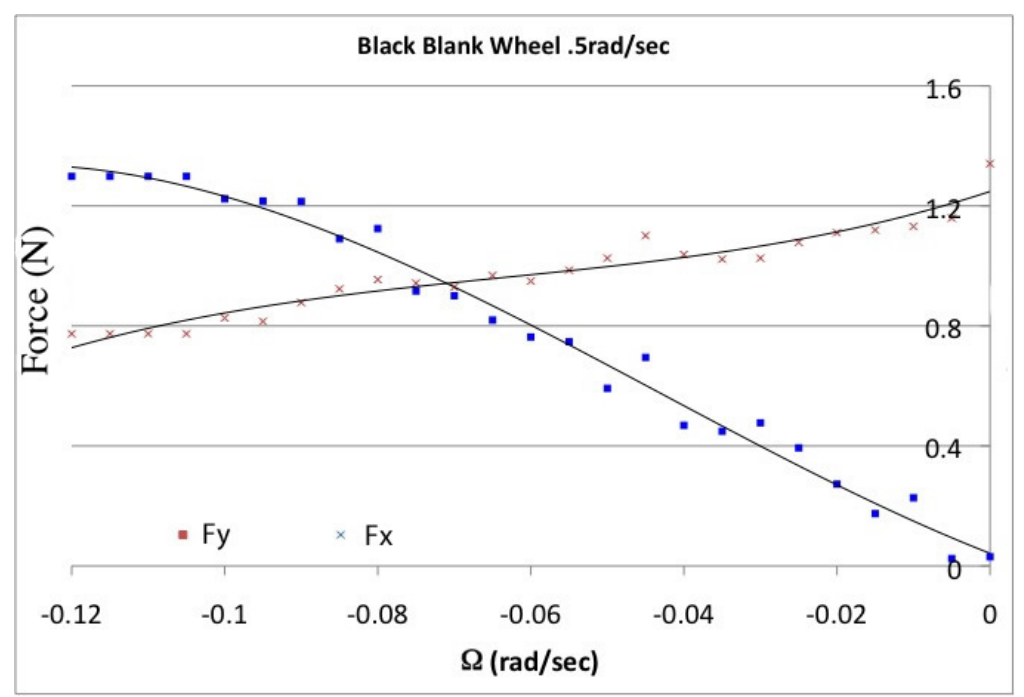

Figure 9: Results for $\omega=.5 \frac{\mathrm{rad}}{\mathrm{sec}}$ 
are given in table 1 along with $\Omega_{\text {Predicted }}$ and percentage error. These results show a definite validation of the SWEET single wheel test within $3 \%$ of actual rover turning performance.

Table 1: $\Omega_{S R 2}$ and $\Omega_{\text {Predicted }}$ results in $\frac{\mathrm{rad}}{\mathrm{sec}}$

\begin{tabular}{llll}
\hline$\omega\left(\frac{\mathrm{rad}}{\mathrm{sec}}\right)$ & $\Omega_{S R 2}\left(\frac{\mathrm{rad}}{\mathrm{sec}}\right)$ & $\Omega_{\text {Predicted }}\left(\frac{\mathrm{rad}}{\mathrm{sec}}\right)$ & Error \\
\hline 0.3 & -.042 & -.042 & $0 \%$ \\
0.4 & -.056 & -.057 & $1.8 \%$ \\
0.5 & -.066 & -.064 & $3.0 \%$ \\
\hline
\end{tabular}

\section{Skid Steer Experiments with Non-Blank Wheels}

In considering a non-blank wheel, particularly a directional patterned wheel such as Figure 11 there is a possibility of a force along the $X$ axis induced by the rotating tread pattern. If the wheel is mounted in the correct orientation then the additional force will benefit the turning efficiency by offsetting the frictional force produced by the turn. The theoretical ideal turning rate for a directional treaded wheel has to include any $V_{x}$ produced by the tread.

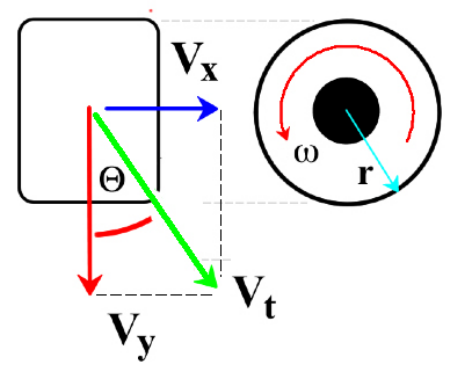

Figure 10: Kinematic explanation of treaded wheel

$$
\begin{gathered}
V_{t}=V_{y} \cos (\Theta)+V_{x} \sin (\Theta) . \\
V_{y}=\omega r K_{y}\left(0 \leq K_{y} \leq 1\right)
\end{gathered}
$$

$K_{y}$ is a measure of wheel slippage in the $Y$ direction, and can be calculated separately by using the $\Omega_{\text {predicted }}$ and geometry values found by:

$$
\begin{gathered}
K_{y}=\frac{\Omega R \cos \Theta}{\omega r} \\
V_{t}=\Omega R .
\end{gathered}
$$




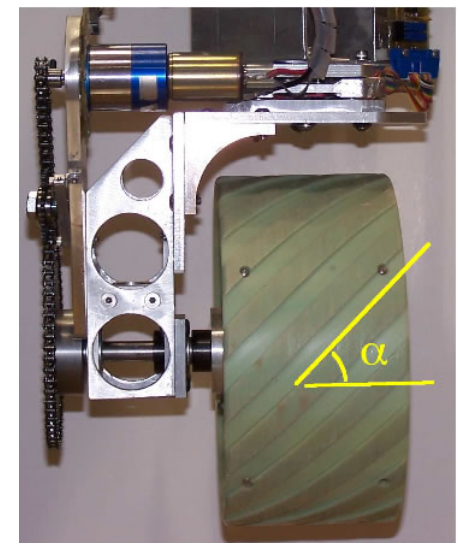

Figure 11: Measuring $\alpha$ on a treaded wheel

$$
\Omega=\frac{1}{R}\left(\omega r K_{y} \cos (\Theta)+V_{x} \sin (\Theta)\right) .
$$

If $\Omega$ was related to the tread design only (such as a bolt screwing into a nut) and ignored any soil interaction $V_{x}$ would be a function of $\omega, \alpha$, and $r$ only. Since this is not reality, $K_{x}$ is used to compensate for the lateral sliding. $K_{y}$ and $K_{x}$ are dependent on the vehicle's geometry and Eq (22), which is the wheel's skid steer turning efficiency based on wheel rotational speed and is defined by:

$$
\begin{gathered}
\frac{\Omega_{\text {predicted }}}{\omega} \\
K_{x}=\frac{\Omega R \sin \Theta \tan \alpha}{\omega r} \\
V_{x}=\frac{\omega r}{\tan (\alpha)} .
\end{gathered}
$$

Which would give:

$$
\Omega=\frac{\omega r}{R}\left(\cos (\Theta) K_{y}+\frac{\sin (\Theta) K_{x}}{\tan (\alpha)}\right) .
$$

Two directional patterned wheels, with diameter of .102 meters (Figure 11), were tested in SWEET. Figures 12 and 13 show the performance of the two oppositely patterned wheels dubbed 'correct' and 'opposite' which correspond to their proper orientation on the vehicle. The opposite wheel can be visualized as trying to screw itself to the right fighting against the turn when placed on the right front side, the correct wheel is trying to screw itself left benefiting the turn. The tests were run on carpet and were designed to simulate the right front side of a vehicle turning in a counter-clock-wise fashion. Figure 13 shows the results of the opposite wheel in that position producing a $\Omega_{\text {predicted }}$ value of 


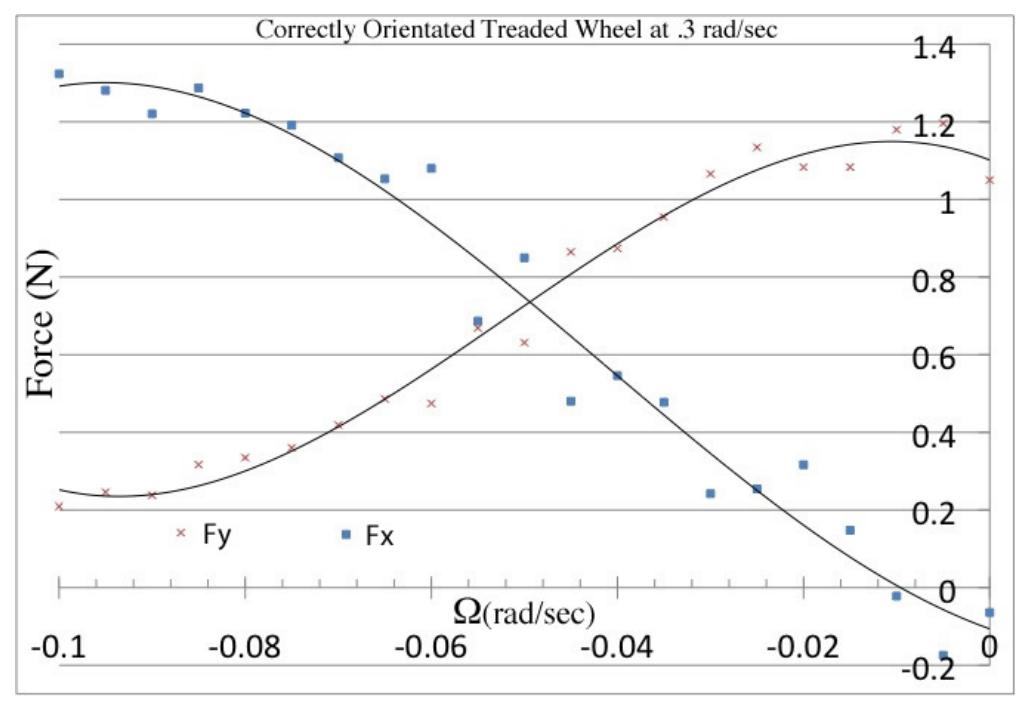

Figure 12: Results for correct treaded wheel rotating at $\omega=.3 \frac{\mathrm{rad}}{\mathrm{sec}}$ in the right front position.

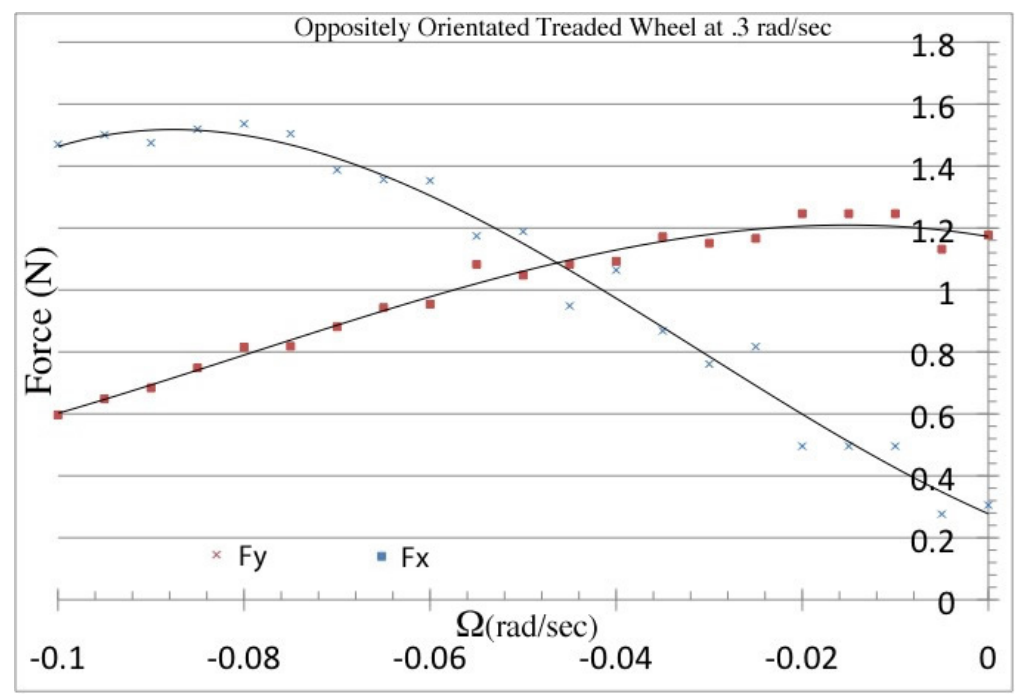

Figure 13: Results for opposite treaded wheel rotating at $\omega=.3 \frac{\mathrm{rad}}{\mathrm{sec}}$ in the right front position.

Table 2: $\Omega_{S R 2}$ and $\Omega_{\text {Predicted }}$ results for treaded wheels at $\omega=.3$

\begin{tabular}{llll}
\hline Wheel & $\Omega_{S R 2}$ & $\Omega_{\text {Predicted }}$ & Error \\
\hline Opposite & -.0410 & -.0417 & $1.7 \%$ \\
Correct & -.0471 & -.0472 & $0.4 \%$ \\
\hline
\end{tabular}


$-.0417 \frac{\mathrm{rad}}{\mathrm{sec}}$ while in Figure 12 the correct wheel gives a $\Omega_{\text {predicted }}$ value of -.0472 $\frac{\mathrm{rad}}{\mathrm{sec}}$ (table 2).

Again the results were compaired to out in house rover with four wheels in both correct and opposite fashion and recorded in table 2. From the table it can be seen that the algorithm was able to predict a full vehicle's skid steer turn rate within $2 \%$ of the experimental value. Also observed is a $15 \%$ increase in turning rate for a correctly orientated wheel over an oppositely orientated wheel, which shows that wheel tread does make a difference in skid steer turning.

\section{5. $F_{x}$ Reduction}

From the force- $\Omega$ graph (Figure 14) it can be seen that as $F_{X}$ (the net force parallel to the wheel axis) is reduced (shown as multiple values of $F_{X 1}$ and $F_{X 2}$ in Figure 14) along all values of $\Omega$ then the vehicle spin rate would be increased and can be explained physically as the effect of reducing the vehicle's friction in the $X$ direction. If the total force $\left(F_{X}\right)$ were reduced enough (by reducing the lateral friction $\left(f_{x}\right)$ or increasing the tread propulsive force $\left.\left(F_{x}\right)\right)$ then it is possible to increase the vehicle's spin rate faster than the driven speed between the wheel and the ground much like a sailboat can tack resulting in speeds faster than the wind that is pushing it. Also from Figure 14 it can be seen that if $F_{Y}$ is decreased then the vehicle spin rate will also decreased. If skid steering performance is to be enhanced then $F_{X}$ must be made independent of $F_{Y}$ and their difference broadened at the equilibrium point. It is not only the reduction of $F_{X}$ independently,but also with out reducing $F_{Y}$, that is an effective design goal.

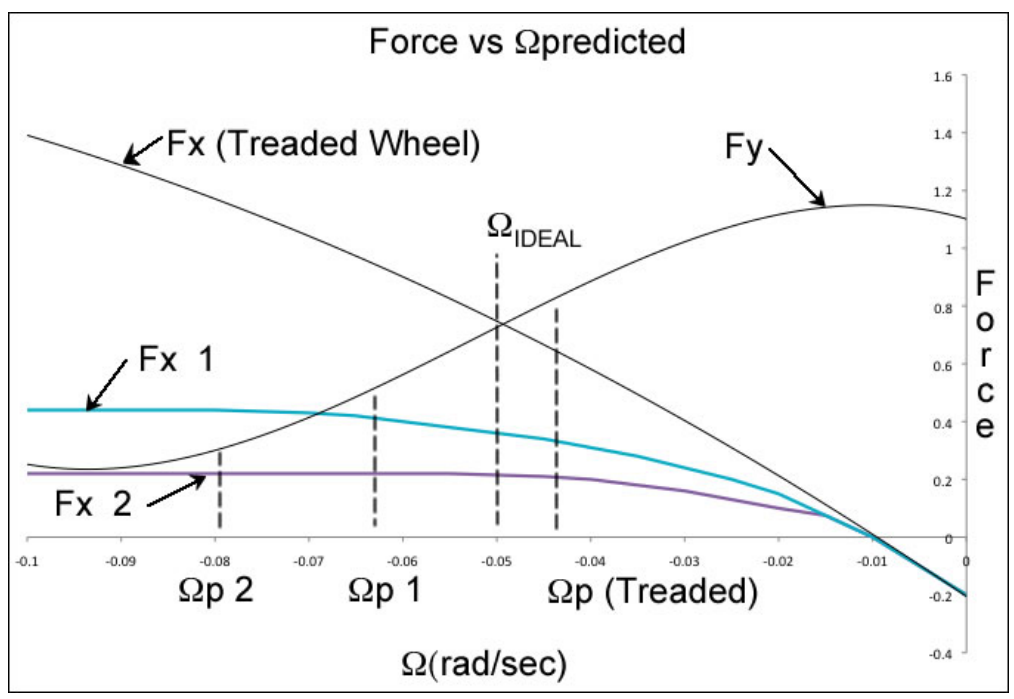

Figure 14: Fx Reduction Example 


\section{1. $F_{X}$ Reduction Experiments}

To demonstrate that an $F_{X}$ reduction can enhance a skid steer turn, tests were run on a blank and an Omni wheel. Omni wheels (Figure 15a) are designed such that they do not restrict motion parallel to their axis. This is done by incorporating numerous passive wheels fixed to the outside circumference. This added degree of freedom adds some interesting possibilities to vehicle motion such as $\langle 15 ; 4 ; 18>$.
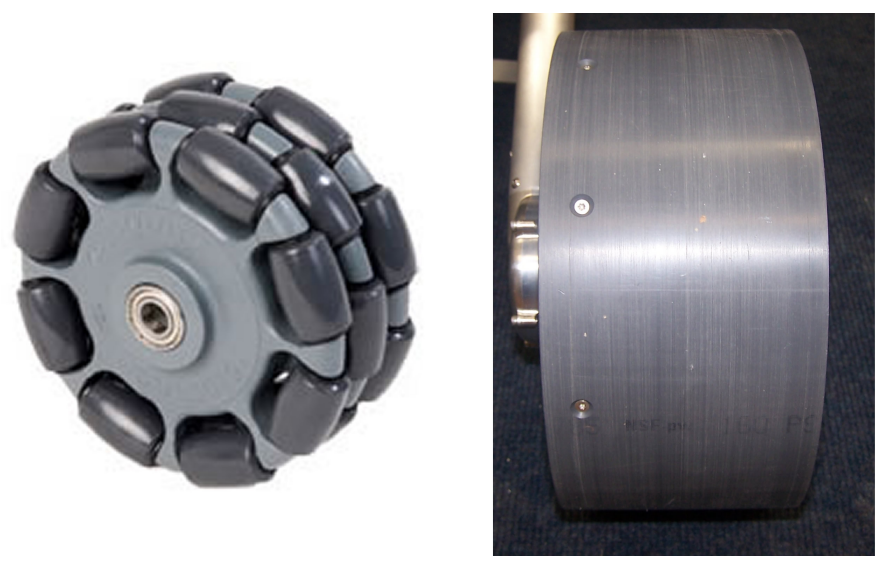

Figure 15: a) Omni-wheel $<3>$ b) Blank Wheel

Both wheels were tested at two different wheel speeds: 0.3 and $0.5 \frac{\mathrm{rad}}{\mathrm{sec}}$ with the range of $\Omega$ from 0 to $0.1 \frac{\mathrm{rad}}{\mathrm{sec}}$ by $.005 \frac{\mathrm{rad}}{\mathrm{sec}}$ increments. Figure 16 shows the $\Omega$ results for the wheel velocity of $0.3 \frac{\mathrm{rad}}{\mathrm{sec}}$ and Figure 17 gives the $\Omega$ results for $0.5 \frac{\mathrm{rad}}{\mathrm{sec}}$ for both wheels. The results show that the Omni wheel outperforms the blank wheel in both tests even though it has a smaller diameter (which gives it a slower turning velocity potential compared to the bigger blank wheel). To normalize the size difference the power usage was also measured and factored in to give a turning power efficiency with units of Eq (26). This comparison further shows the difference of the two wheels' skid steering ability. For the vehicle geometry tested, the velocity for an Ackerman vehicle is 0.0433 for $\omega=0.3$ and 0.0722 for $\omega=0.5$. As can be seen, the Omni wheel exceeds these values which means that a skid steering vehicle fitted with four Omni wheels would turn better than a vehicle the same size with a steerable wheel suspension, but only if the omni wheels are arranged so the vehicle has no lateral stability.

$$
\frac{\Omega}{\text { Power }}=\frac{\text { rad }}{\text { Wattsec }}
$$




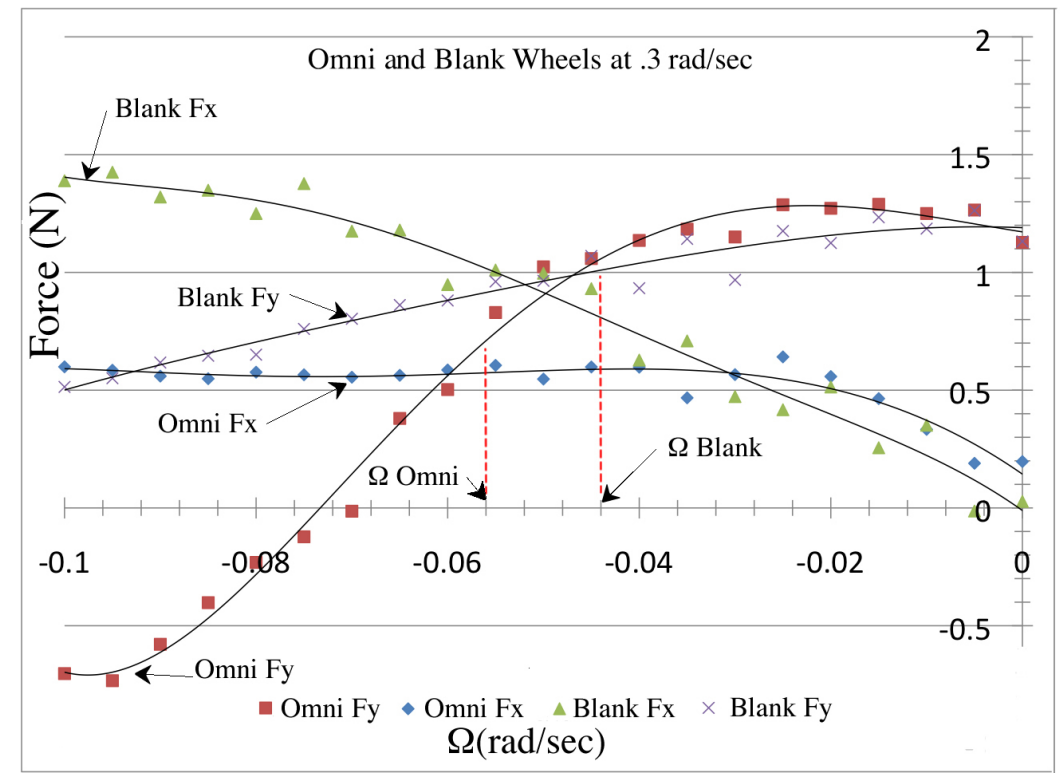

Figure 16: Results for Omni and blank wheel spinning at .3 $\frac{\mathrm{rad}}{\mathrm{sec}}$

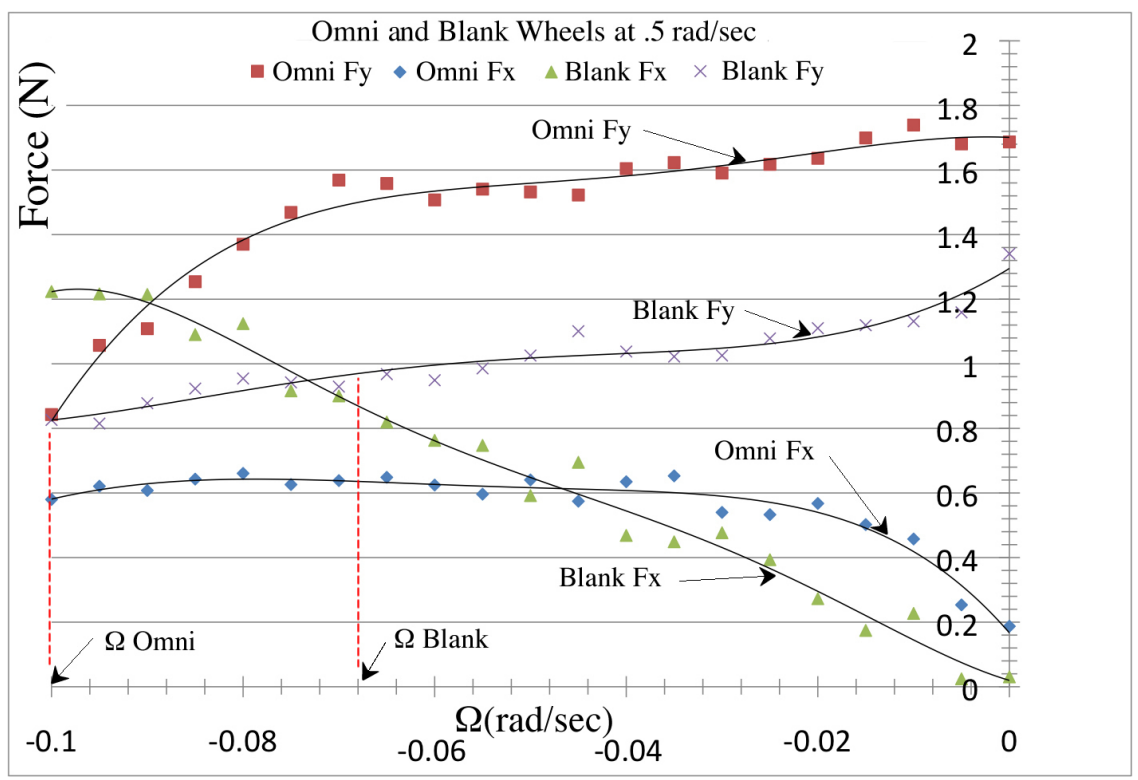

Figure 17: Results for Omni and blank wheel spinning at .5 $\frac{\mathrm{rad}}{\mathrm{sec}}$ 
Table 3: $\Omega_{S R 2}$ and $\Omega_{\text {Predicted }}$ results for Omni wheels

\begin{tabular}{|l|l|l|l|l|l|l|}
\hline$\omega \frac{\mathrm{rad}}{\mathrm{sec}}$ & $\begin{array}{l}\text { Omni } \\
\Omega_{\text {Predicted }}\end{array}$ & $\begin{array}{l}\text { Blank } \\
\Omega_{\text {Predicted }}\end{array}$ & $\begin{array}{l}\% \\
\text { Difference }\end{array}$ & $\begin{array}{l}\text { Omni } \\
\frac{\Omega}{\text { Power }}\end{array}$ & $\begin{array}{l}\text { Blank } \\
\frac{\Omega}{\text { Power }}\end{array}$ & $\begin{array}{l}\% \\
\text { Difference }\end{array}$ \\
\hline .3 & -.058 & -.042 & $38 \%$ & .003 & .002 & $100 \%$ \\
.5 & -.105 & -.064 & $64 \%$ & .005 & .002 & $150 \%$ \\
\hline
\end{tabular}

\section{Conclusions}

This paper discusses and demonstrates a method that allows the results from a single wheel test to be used to predict turning efficiency for a full assembly skid steer vehicle. Three different wheels were tested and predicted turn rates were within $3 \%$ of full assembly tests. It can be seen in the few tests done that the tread design does make a performance difference, and in skid steering can either hurt or help the turning efficiency depending on the tread design.

Also discussed and demonstrated is a method of increasing skid steering efficiency by independently altering the frictional components of a wheel. An Omni wheel was tested in a simulated skid steer turn and compared to a blank wheel in the same turn. As predicted the Omni wheel performed much better than the blank wheel and even turned the vehicle faster than if it had a steerable wheel suspension. Omni Wheels with orthogonal wheels are impractical on vehicles of this type but do show that reducing $F_{X}$ independently of $F_{Y}$ can enhance a vehicle's skid steer turn. Other factors would need to be weighed and factored in depending on the vehicle's mission or directive. Such as draw-bar pull, lateral sliding, and straight line efficiency.

Future work will be to test on sand and other terrain evaluate other interesting wheel types, and iterate tread design on conventional wheels to better ascertain a wheel's performance on different media all of which without the cost and time of full assembly tests. Also other work would include expanding into skid steer turns that don't only rotate about the center axis of the vehicle.

\section{References}

[1] Said Al-Milli, Lakmal D. Seneviratne, and Kaspar Althoefer. Track-terrain modelling and traversability prediction for tracked vehicles on soft terrain. Journal of Terramechanics, 47(3):151 - 160, 2010.

[2] C.A. Brooks, K.D. Iagnemma, and S. Dubowsky. Visual wheel sinkage measurement for planetary rover mobility characterization. Auton. Robots (Netherlands), 21(1):55 - 64, 2006/08.

[3] Kornylak Corp. Omniwheel, heavy duty and lightweight multi-directional wheels for conveyors, manual and powered transfer systems, and nonpowered and powered robot wheels. Technical report, 400 Heation St Hamilton, Ohio 45011. 
[4] Jefferey Farnam. Four-wheel drive wheel-chair with compound wheels,". Technical Report 4,823,900, United States Patent Office, 1989.

[5] Daniel Flippo. Design of rover wheel testing apparatus. In ISAIRAS, 2008.

[6] Daniel Flippo and David Miller. Suspension and wheel evaluation and experimentation testbed for planetary rovers. International Journal of Mechatronics and Automation, 1(1):29-37, 2011.

[7] G. Ishigami, A. Miwa, K. Nagatani, and K. Yoshida. Terramechanics-based model for steering maneuver of planetary exploration rovers on loose soil. J. Field Robot. (USA), 24(3):233 - 50, 2007/03/.

[8] D. King-Hele. Erasmus darwin's improved design for steering carriages and cars. Notes and Records of the Royal Society, 56:41-62, 2002.

[9] Andrew J. LePage. Ahead of their time: The soviet lunar program of the seventies ahead of their time: The soviet lunar program of the seventies part i. The Electronic Journal of the Astronomical Society of the Atlantic, 7(1), January 1996.

[10] Proceedings of the Institution of Mechanical Engineers, Part D: Journal of Automobile Engineering. A general theory for skid steering of tracked vehicles on firm ground, volume 215. Professional Engineering Publishing, 2001.

[11] M. A. Ravine, J. F. Bell III, M. C. Malin, and D. P. Miller. Semi-autonomous rover operations: A mars technology program semiautonomous rover operations: A mars technology program demonstration. Lunar and Planetary Science XXXVI, 2005.

[12] Matthew J. Roman. Design and analysis of a four wheeled planetary rover. Master's thesis, University of Oklahoma, August 2005.

[13] S.Michaud, L.Richter, T.Thueer, A.Gibbesch, T.Huelsing, N. Schmitz, S. Weiss, A.Krebs, N.Patel, L.Joudrier, R.Siegwart, B.Schäfer, and A.Ellery. Rover chassis evaluation and design optimisation using the rcet, 2006 .

[14] Standard Testing Laboratories, 1845 Harsh Ave. S.E. P.O. Box 758, Massillon, Ohio 44648-0758 USA. Radial Fatigue Two-Position Tire and Wheel Test Machine.

[15] M. Wada. A 4wd omnidirectional wheelchair with a chair tilting mechanism for enhancing step climbing capability. pages 474-479, March 2008.

[16] Hongpeng Wang, Junjie Zhang, Jingang Yi, Dezhen Song, S. Jayasuriya, and Jingtai Liu. Modeling and motion stability analysis of skid-steered mobile robots. In Robotics and Automation, 2009. ICRA '09. IEEE International Conference on, pages 4112-4117, 2009. 
[17] Jingang Yi, Hongpeng Wang, Junjie Zhang, Dezhen Song, S. Jayasuriya, and Jingtai Liu. Kinematic modeling and analysis of skid-steered mobile robots with applications to low-cost inertial-measurement-unit-based motion estimation. Robotics, IEEE Transactions on, 25(5):1087-1097, 2009.

[18] A. Zobova and Ya Tatarinov. Free and controlled motions of an omniwheel vehicle. Moscow University Mechanics Bulletin, 63(6):146-150, December 2008. 